Internat. J. Math. \& Math. Sci.

Vol. 22, No. 1 (1999) 91-95

S 0161-1712〈99 $22091-1$

(c) Electronic Publishing House

\title{
ON AZUMAYA GALOIS EXTENSIONS AND SKEW GROUP RINGS
}

\section{GEORGE SZETO}

(Received 6 November 1997 and in revised form 22 December 1997)

\begin{abstract}
Two characterizations of an Azumaya Galois extension of a ring are given in terms of the Azumaya skew group ring of the Galois group over the extension and a Galois extension of a ring with a special Galois system is determined by the trace of the Galois group.
\end{abstract}

Keywords and phrases. Azumaya algebras, Galois extensions, $H$-separable extensions, skew group rings.

1991 Mathematics Subject Classification. 16S30, 16W20.

1. Introduction. Let $S$ be a ring with $1, G$ a finite automorphism group of $S$ of order $n$ for some integer $n$ invertible in $S, S^{G}$ the subring of the elements fixed under each element in $G, C$ the center of $S$, and $S^{*} G$ the skew group ring of $G$ over $S$. In [3] and [2], $S$ is called an Azumaya Galois extension of $S^{G}$ if it is a $G$-Galois extension of $S^{G}$ which is an Azumaya $C^{G}$-algebra. It was shown that $S$ is an Azumaya Galois extension if and only if $S^{*} G$ is an Azumaya $C^{G}$-algebra. The purpose of the present paper is to give two more characterizations of an Azumaya Galois extension in terms of the Azumaya skew group ring $S^{*} G$. We show that $S$ is an Azumaya $G$-Galois extension if and only if $S^{*} G$ is an Azumaya algebra over its center $Z$, a $G^{\prime}$-Galois extension with an inner Galois group $G^{\prime}$ induced by the elements of $G$, and $Z G$ is a finitely generated projective $C^{G}$-module of rank $n$. Moreover, for the skew group ring $S^{*} G$, where $S$ is a separable $C^{G}$-algebra, an expression of the commutator subring of $C$ in $S^{*} G$ is obtained by using $S$ and its commutator subring in $S^{*} G$. Furthermore, let $H$ be a normal subgroup of $G$, $K$ the commutator subgroup of $H$ in $G$, and $H^{\prime}$ the inner automorphism group of $S^{*} G$ induced by the elements of $H\left(K^{\prime}\right.$ and $(G / H)^{\prime}$ are similarly defined). Then, it is shown that $\left(S^{*} G\right)^{K^{\prime}}$ is a $(G / K)^{\prime}$-Galois extension with a Galois system $\left\{m^{-1} g_{j}, g_{j}^{-1} / g_{j}\right.$ in $\left.H\right\}$ if and only if $\operatorname{Tr}_{G^{\prime}}\left(g_{i}\right)=0$ for each $g_{i}$ not in $K$, where $m$ is the order of $H$ for some integer $m$ and $\operatorname{Tr}_{G^{\prime}}\left(g_{i}\right)$ is the trace of $G^{\prime}$ at $g_{i}$.

2. Preliminaries. Throughout, let $S$ be a ring with $1, G=\left\{g_{1}, \ldots, g_{n}\right\}$ for some integer $n$ invertible in $S, C$ the center of $S, S^{G}$ the subring of the elements fixed under each element in $G$, and $S^{*} G$ the skew group ring of $G$ over $S$. Let $B$ be a subring of a ring $A$. We call $A$ a separable extension of $B$ if there exist $\left\{a_{i}, b_{i}\right\}$ in $A$, $i=1, \ldots, m$ for some integer $m$, such that $\sum a_{i} b_{i}=1$ and $\sum a a_{i} \otimes b_{i}=\sum a_{i} \otimes b_{i} a$ for all $a$ in $A$, where $\otimes$ is over $B$ and $\left\{a_{i}, b_{i}\right\}$ is called a separable system for $A$. An Azumaya algebra is a separable extension over its center. A ring $A$ is called an $H$-separable extension of $B$ if $A \otimes A$ is a direct summand of a finite direct sum of $A$ as an $A$-bimodule, where $\otimes$ over $B$. Denote the commutator subring of $B$ in $A$ by 
$V_{A}(B)$. An $H$-separable extension $A$ over $B$ is equivalent to the existence of an $H$ separable system $\left\{d_{i}\right.$ in $V_{A}(B) ; \sum\left(x_{i j} \otimes y_{i j}\right)$ in $\left.V_{A \otimes A}(A)\right\}, j=1, \ldots, u$ and $i=1, \ldots, v$ for some integers $u$ and $v$ such that $\sum d_{i}\left(\sum\left(x_{i j} \otimes y_{i j}\right)\right)=1 \otimes 1, i=1, \ldots, v$ and $j=1, \ldots, u$. The ring $S$ is called a $G$-Galois extension of $S^{G}$ if there exist $\left\{c_{i}, d_{i}\right.$ in $S, i=$ $1, \ldots, k$ for some integer $k\}$ such that $\sum c_{i} d_{i}=1$ and $\sum a_{i} g_{j}\left(b_{i}\right)=0$ for each $g_{j} \neq 1$, where $\left\{c_{i}, d_{i}\right\}$ is called a $G$-Galois system for $S$. It is well known that an Azumaya algebra is an $H$-separable extension and that an $H$-separable extension is a separable extension. A skew group $\operatorname{ring} S^{*} G$ is a ring with a free basis $\left\{g_{i}\right\}$ over $S$ such that $g_{i} s=\left(g_{i}(s)\right) g_{i}$ for each $g_{i}$ in $G$ and $s$ in $S$. We denote the center of $S^{*} G$ by $Z$, the inner automorphism group of $S^{*} G$ induced by the elements of the subgroup $H$ of $G$ by $H^{\prime}$ $\left(=\left\{g^{\prime} / g^{\prime}(x)=g x g^{-1}\right.\right.$ for $g$ in $H$ and all $x$ in $\left.\left.S^{*} G\right\}\right)$, and the commutator subgroup of $H$ in $G$ by $V_{G}(H)$.

3. Skew group rings. In this section, keeping the notations of Section 2, we give two characterizations of an Azumaya Galois extension and an expression of the commutator subring of $C$ in $S^{*} G$ when $S$ is a separable $C^{G}$-algebra.

THEOREM 3.1. The following statements are equivalent:

(i) $S$ is an Azumaya Galois extension,

(ii) $S^{*} G$ is an Azumaya $Z$-algebra and $S$ satisfies the double centralizer property in $S^{*} G$, and

(iii) $S^{*} G$ is an Azumaya $Z$-algebra and a $G^{\prime}$-Galois extension of $\left(S^{*} G\right)^{G^{\prime}}$, and $Z G$ is a finitely generated and projective $C^{G}$-module of rank $n$.

Proof. (i) $\Rightarrow$ (ii). Since $S$ is an Azumaya Galois extension, $S^{*} G$ is an Azumaya $C^{G_{-}}$ algebra (that is, $Z=C^{G}$ ) and $S^{*} G$ is an $H$-separable extension of $S$ [3, Thm. 3.1]. Noting that $S$ is a direct summand of $S^{*} G$ as a left $S$-module, we conclude that $V_{S^{*} G}\left(V_{S^{*} G}(S)\right)=S$ [6, Prop. 1.2].

(ii) $\Rightarrow$ (i). Since $V_{S{ }^{*} G}\left(V_{S{ }_{G} G}(S)\right)=S, Z$ is contained in $S$; and so $Z$ is contained in $C$. But then $Z=C^{G}$. This implies that $S^{*} G$ is an Azumaya $C^{G}$-algebra by (ii). Thus, $S$ is an Azumaya Galois extension [3, Thm. 3.1].

(i) $\Rightarrow$ (iii). Since the restriction of $G^{\prime}$ to $S$ is $G, S^{*} G$ is a $G^{\prime}$-Galois extension of $\left(S^{*} G\right)^{G^{\prime}}$ with the same Galois system as $S$ (for $S$ is $G$-Galois). Also, by hypothesis, $S$ is an Azumaya Galois extension, so $S^{*} G$ is an Azumaya $C^{G}$-algebra [3, Thm. 3.1]. Moreover, since $Z=C^{G}, Z G$ is a free $Z$-module of rank $n$.

(iii) $\Rightarrow$ (i). Since $S^{*} G$ is a $G^{\prime}$-Galois extension of $\left(S^{*} G\right)^{G^{\prime}}$ with an inner Galois group $G^{\prime}$, it is an $H$-separable extension of $\left(S^{*} G\right)^{G^{\prime}}$ [7, Cor. 3]. But $n$ is a unit in $S$, so $V_{S^{*} G}\left(\left(S^{*} G\right)^{G^{\prime}}\right)$ is a separable $Z$-algebra and a finitely generated and projective $Z$ module of rank $n$ [7, Prop. 4]. Moreover, $S^{*} G$ is a $G^{\prime}$-Galois extension of $\left(S^{*} G\right)^{G^{\prime}}$, so it is finitely generated and projective $\left(S^{*} G\right)^{G^{\prime}}$-module. Since $n$ is a unit in $S, Z G$ is a separable $Z$-algebra. But then $V_{S^{*} G}\left(\left(S^{*} G\right)^{G^{\prime}}\right)=V_{S * G}\left(V_{S^{*} G}(Z G)\right)=Z G$ by the commutator theorem for Azumaya algebras [4, Thm. 4.3]. Therefore, $Z G$ is a finitely generated and projective $Z$-module of rank $n$ [1, Prop. 4]. From the fact that there are $n$ elements $\left\{g_{i}\right\}$ of $G$ as generators of $Z G$, it is not difficult to show that $\left\{g_{i}\right\}$ are free over $Z$. Hence, $Z$ is a finitely generated and projective $C^{G}$-module. Thus, the rank of $Z G$ over $C^{G}$ is a product of the rank of $Z G$ over $Z$ and the rank of $Z$ over $C^{G}$; that is, 
$n=n$ (rank of $Z$ over $C^{G}$ ). This implies that $Z=C^{G}$. Therefore, $S^{*} G$ is an Azumaya $C^{G}$-algebra; and so $S$ is an Azumaya Galois extension [3, Thm. 3.1].

COROLLARY 3.2. Let $S$ be a separable $C^{G}$-algebra. If $V_{S{ }_{G}}(S)$ is a $G^{\prime \prime}$-Galois extension, where $G^{\prime \prime}$ is the inner automorphism group of $V_{S^{*} G}(S)$ induced by and isomorphic with $G$, then $S$ is an Azumaya Galois algebra.

Proof. Since $V_{S^{*} G}(S)$ is a $G^{\prime \prime}$-Galois extension, there exists a $G^{\prime \prime}$-Galois system $\left\{c_{i}, d_{i}\right.$ in $\left.V_{S^{*} G}(S) \mid i=1, \ldots, k\right\}$ for $V_{S^{*} G}(S)$. Then, it is straightforward to check that $\left\{c_{j} ; \sum g_{j} d_{i} \otimes g_{j}^{-1}, i=1, \ldots, k\right.$ and $j=1, \ldots, m$ for some integers $k$ and $\left.m\right\}$ is an $H$-separable system for $S^{*} G$ over $S$ [1, Thm. 1]. Hence, $S$ satisfies the double centralizer property in $S^{*} G$ [7, Prop. 1.2]. Moreover, $n$ is a unit in $S$, so $S^{*} G$ is a separable extension of $S$. By hypothesis, $S$ is a separable $C^{G}$-algebra, so $S^{*} G$ is a separable $C^{G}$-algebra by the transitivity of separable extensions. But then $S^{*} G$ is an Azumaya $Z$-algebra. Therefore, $S$ is an Azumaya Galois extension by Theorem 3.1.

For the skew group ring $S^{*} G$ of $G$ over a separable $C^{G}$-algebra $S$, we next give an expression of $V_{S{ }^{*} G}(C)$ in terms of $S$ and $V_{S{ }^{*} G}(S)$ (for more about $V_{S^{*} G}(S)$, see [1]).

THEOREM 3.3. If $S$ is a separable $C^{G}$-algebra, then

(i) $C Z$ is a commutative separable subalgebra of $S^{*} G$ and

(ii) $S Z, V_{S^{*} G}(S)$, and $V_{S^{*} G}(C)$ are Azumaya $C Z$-algebras contained in $S^{*} G$, such that $V_{S * G}(C) \cong S Z \otimes V_{S{ }^{*} G}(S)$, where $\otimes$ is over $C Z$.

Proof. (i) Since $S$ is a separable $C^{G}$-algebra, $C$ is also a separable $C^{G}$-algebra. Hence, $C \otimes Z$ is a separable $Z$-algebra, where $\otimes$ is over $C^{G}$; and so the homomorphic image $C Z$ of $C \otimes Z$ is also a separable $Z$-algebra. Clearly, $C Z$ is commutative.

(ii) Since $n$ is a unit in $S, S^{*} G$ is a separable $S$-extension. Hence, $S^{*} G$ is a separable $C^{G_{-}}$algebra by the transitivity of separable extensions; and so $S^{*} G$ is an Azumaya $Z$ algebra. But then $V_{S^{*} G}(C Z)$ is a separable subalgebra of $S^{*} G$ such that $V_{S{ }^{*}}\left(V_{S^{*} G}(C Z)\right)$ $=C Z$ [4, Thm. 4.3] (for $C Z$ is a separable subalgebra of $S^{*} G$ by (i)). This implies that the center of $V_{S{ }^{*} G}(C Z)$ is $C Z$. Thus, $V_{S * G}(C Z)$ is an Azumaya $C Z$-algebra. By hypothesis again, $S$ is a separable $C^{G}$-algebra, so it is an Azumaya $C$-algebra. Hence, $S \otimes C Z$ is an Azumaya $C Z$-algebra, where $\otimes$ is over $C$. Thus, $S Z$ is also an Azumaya $C Z$-algebra. Noting that $S Z \subset V_{S^{*} G}(C Z)$, we conclude that $V_{S{ }^{*} G}(C Z) \cong S Z \otimes V_{S^{*} G}(S Z)$, where $\otimes$ is over $C Z$ [7, Thm. 4.3]. Moreover, since $V_{S * G}(C Z)=V_{S^{*} G}(C)$ and $V_{S * G}(S Z)=V_{S^{*} G}(S)$, we conclude that $V_{S * G}(C) \cong S Z \otimes V_{S * G}(S)$, where $\otimes$ is over $C Z$.

By [3, Thm. 3.1], if $S$ is an Azumaya Galois extension, then $S^{*} G$ is an Azumaya $C^{G_{-}}$ algebra (that is, $Z=C^{G}$ ) and $S$ is a separable $C^{G}$-algebra. Thus, we have the following result.

COROLLARY 3.4. If $S$ is an Azumaya Galois extension, then $V_{S{ }^{*} G}(C) \cong S \otimes V_{S * G}(S)$ as Azumaya $C$-algebras, where $\otimes$ is over $C$ such that $V_{S^{*} G}(C)$ is a $G^{\prime}$-Galois extension of $V_{S^{*} G}(C G)$.

Proof. By the above remark, it suffices to show that $V_{S^{*} G}(C)$ is a $G^{\prime}$-Galois extension of $V_{S^{*} G}(C G)$. In fact, since $S$ is a $G$-Galois extension and $S \subset V_{S{ }^{*} G}(C), V_{S * G}(C)$ is a $G^{\prime}$-Galois extension with the same Galois system as $S$ by noting that $V_{S^{*} G}(C)$ is 
$G^{\prime}$-invariant (for $G$ is the restriction of $G^{\prime}$ to $S$ ). Moreover, it is clear that $\left(V_{S^{*} G}(C)\right)^{G^{\prime}}=$ $V_{S * G}(C G)$.

4. A Galois system. It is well known that $\left\{n^{-1} g_{i}, g_{i}^{-1} \mid g_{i}\right.$ in $\left.G\right\}$ is a separable system for a separable group ring $R G$ over a ring $R$ with 1 , where $G=\left\{g_{i} \mid i=1, \ldots, n\right\}$ for some integer $n$ invertible in $R$, for a separable skew group ring $S^{*} G$ over $S$ and for a separable projective group ring $R G_{f}$ over $R$ as defined in [9]. In this section, we give an equivalent condition for $\left(S^{*} G\right)^{K^{\prime}}$ to have a $(G / K)^{\prime}$-Galois system similar to the above separable system for a normal subgroup $K$ of $G$.

THEOREM 4.1. Let $H$ be a normal subgroup of $G$ and $V_{G}(H)=K$. Then

(i) $K$ is a normal subgroup of $G$ and

(ii) $\operatorname{Tr}_{H^{\prime}}\left(g_{i}\right)=0$ for each $g_{i}$ not in $K$ if and only if $\left(S^{*} G\right)^{K^{\prime}}$ is a $(G / K)^{\prime}$-Galois extension of $\left(S^{*} G\right)^{G^{\prime}}$ with a Galois system $\left\{m^{-1} g_{j}, g_{j}^{-1} \mid g_{j}\right.$ in $\left.H\right\}$, where $m$ is the order of $H$.

Proof. (i) We want to show that $g_{i} K g_{i}^{-1} \subset K$ for each $g_{i}$ in $G$. For any $x$ in $K$ and $y$ in $H, g_{i} x g_{i}^{-1} y=g_{i} x g_{i}^{-1} y g_{i} g_{i}^{-1}=g_{i} x z g_{i}^{-1}$, where $z=g_{i}^{-1} y g_{i}$. Since $H$ is normal in $G, z$ is in $H$. Hence, $x z=z x$. But then $g_{i} x g_{i}^{-1} y=g_{i} x z g_{i}^{-1}=g_{i} z x g_{i}^{-1}=$ $g_{i} g_{i}^{-1} y g_{i} x g_{i}^{-1}=y g_{i} x g_{i}^{-1}$. This implies that $g_{i} x g_{i}^{-1}$ is in $K$. Thus, $K$ is normal in $G$.

(ii) Assume that $\operatorname{Tr}_{H^{\prime}}\left(g_{i}\right)=0$ for each $g_{i}$ not in $K$. Then $\sum g_{j} g_{i} g_{j}^{-1}=0$, where $H=$ $\left\{g_{j} \mid j=1, \ldots, m\right.$ for some integer $\left.m\right\}$; that is, $\sum g_{j} g_{i} g_{j}^{-1} g_{i}^{-1} g_{i}=\sum g_{j}\left(\left(g_{i}\right)^{\prime}\left(g_{j}^{-1}\right)\right) g_{i}$ $=0, j=1, \ldots, m$. Hence, $\left(m^{-1}\right) \sum g_{j}\left(\left(g_{i}\right)^{\prime}\left(g_{j}^{-1}\right)\right)=0$ for each $g_{i}$ not in $K$. Clearly, for each $g_{i}$ in $K,\left(m^{-1}\right) \sum g_{j}\left(\left(g_{i}\right)^{\prime}\left(g_{j}^{-1}\right)\right)=1$. Thus, $\left\{m^{-1} g_{j}, g_{j}^{-1} \mid g_{j}\right.$ in $\left.H\right\}$ is a $(G / K)^{\prime}$ Galois system for $\left(S^{*} G\right)^{K^{\prime}}$ (for $H \subset\left(S^{*} G\right)^{K^{\prime}}$ ), where $m$ is the order of $H$.

Conversely, $\left(m^{-1}\right) \sum g_{j}\left(\left(g_{i}\right)^{\prime}\left(g_{j}^{-1}\right)\right)=0$ for each $g_{i}$ not in $K$, so $\sum g_{j} g_{i} g_{j}^{-1} g_{i}^{-1}=0$. Hence, $\sum g_{j} g_{i} g_{j}^{-1}=0$; that is, $\operatorname{Tr}_{H^{\prime}}\left(g_{i}\right)=0$ for each $g_{i}$ not in $K$.

We derive the following corollaries.

COROLLARY 4.2. $S^{*} G$ has a $(G / K)^{\prime}$-Galois system $\left\{n^{-1} g_{i}, g_{i}^{-1} \mid g_{i}\right.$ in $\left.G\right\}$, where $K$ is the center of $G$, if and only if $\operatorname{Tr}_{G^{\prime}}\left(g_{i}\right)=0$ for each $g_{i}$ not in $K$.

Proof. Let $H$ be $G$. Then $K=$ the center of $G$; and so the corollary follows immediately from the theorem.

COROLlary 4.3. $S^{*} G$ has a $G^{\prime}$-Galois system $\left\{n^{-1} g_{i}, g_{i}^{-1} \mid g_{i}\right.$ in $\left.G\right\}$ if and only if $\operatorname{Tr}_{G^{\prime}}\left(g_{i}\right)=0$ for each $g_{i} \neq 1$.

Proof. This is the case of the theorem that the center of $G$ is trivial.

We derive an equivalent condition for a Galois subring of $S^{*} G$ arising from a $G^{\prime}$ invariant subring.

COROLLARY 4.4. Let $A$ be $a G^{\prime}$-invariant subring of $S^{*} G$ and $H=\left\{g_{i}\right.$ in $G \mid g_{i}(a)=$ a for each $a$ in $A$ \}. Then

(i) $H$ is normal in $G$ and

(ii) Denoting $\left(S^{*} G\right)^{H^{\prime}}$ by $B$ and $V_{G}(H)$ by $K, \operatorname{Tr}_{K^{\prime}}\left(g_{i}\right)=0$ for each $g_{i}$ not in $H$ if and only if $\left\{m^{-1} g_{j}, g_{j}^{-1} \mid g_{j}\right.$ in $\left.K\right\}$ is a $(G / H)^{\prime}$-Galois system for $B$, where $m$ is the 
order of $K$.

Proof. Part (i) is straightforward and part (ii) follows immediately from Theorem 4.1.

We conclude the present paper with an example of an Azumaya skew group ring $S^{*} G$ which is a $G^{\prime}$-Galois extension such that the rank of $Z G$ over $C^{G}$ is not $n$ (see Theorem 3.1(iii)). Hence, $S$ is not an Azumaya Galois extension by Theorem 3.1.

Let $R$ be the real field, $S=R[i, j, k]$ the quaternion algebra over $R$, and $G=\{1, g \mid$ $g(x)=i x(i)^{-1}$ for each $x$ in $\left.S\right\}$. Then

(1) $S$ is a $G$-Galois extension with a Galois system $\left\{2^{-1}, 2^{-1} j ; 1,-j\right\}$. Hence, $S^{*} G$ is a $G^{\prime}$-Galois extension with the same Galois system.

(2) Since $S^{*} G$ is a separable extension of $S$ and $S$ is an Azumaya $R$-algebra, $S^{*} G$ is a separable $R$-algebra. Hence, $S^{*} G$ is an Azumaya $Z$-algebra.

(3) The center $Z$ of $S^{*} G$ is $(R+R i)$ by direct computation.

(4) $Z G$ is free over $Z$ by direct verification.

(5) $C=R$ and $C^{G}=C=R$.

(6) $Z$ is a free $R$-module of rank 2 and $Z G$ is a free $C^{G}$-module of rank $4(\neq 2=$ the order of $G$ ), so one of the three conditions in Theorem 3.1(iii) does not hold.

ACKNOWLEDGEMENT. This work was supported by the Caterpillar Fellowship at Bradley University during the author's sabbatical leave in Fall, 1997. The present paper was revised according to the suggestions of the referee. The author would like to thank the referee for his valuable suggestions.

\section{REFERENCES}

[1] R. Alfaro and G. Szeto, The centralizer on $H$-separable skew group rings, Rings, extensions, and cohomology (New York), Lecture Notes in Pure and Appl. Math., vol. 159, Marcel Dekker, Inc., 1994, Basal Hong Kong, pp. 1-7. MR 95g:16027. Zbl 812.16038.

[2] _ Skew group rings which are Azumaya, Comm. Algebra 23 (1995), no. 6, 2255-2261. MR 96b:16027. Zbl 828.16030.

[3] _ On Galois extensions of an Azumaya algebra, Comm. Algebra 25 (1997), no. 6, 1873-1882. CMP 97 11. Zbl 890.16017.

[4] F. DeMeyer and E. Ingraham, Separable algebras over commutative rings, Lecture Notes in Mathematics, vol. 181, Springer-Verlag, New York, 1971. MR 43\#6199. Zbl 215.36602.

[5] F. R. DeMeyer, Some notes on the general Galois theory of rings, Osaka J. Math. 2 (1965), 117-127. MR 32\#128. Zbl 143.05602.

[6] K. Sugano, Note on semisimple extensions and separable extensions, Osaka J. Math. 4 (1967), 265-270. MR 37\#1412. Zbl 199.07901.

[7] _ On a special type of Galois extensions, Hokkaido Math. J. 9 (1980), no. 2, 123-128. MR 82c:16036. Zbl 467.16005.

[8] G. Szeto, Separable Subalgebras of a Class of Azumaya Algebras, to appear in Internat. J. Math. Math. Sci.

[9] G. Szeto and Y. F. Wong, On Azumaya projective group rings, Azumaya algebras, actions, and modules, Contemp. Math., vol. 124, Amer. Math. Soc., 1992, pp. 251-256. MR 92m:16024. Zbl 749.16013. 


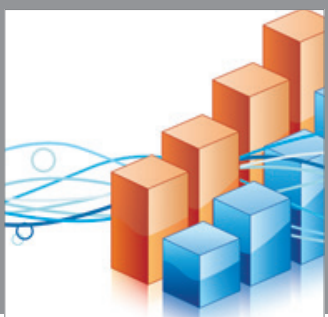

Advances in

Operations Research

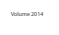

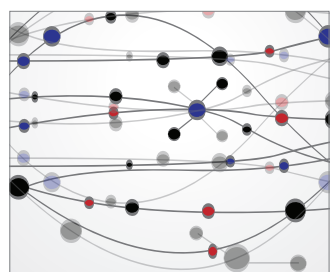

\section{The Scientific} World Journal
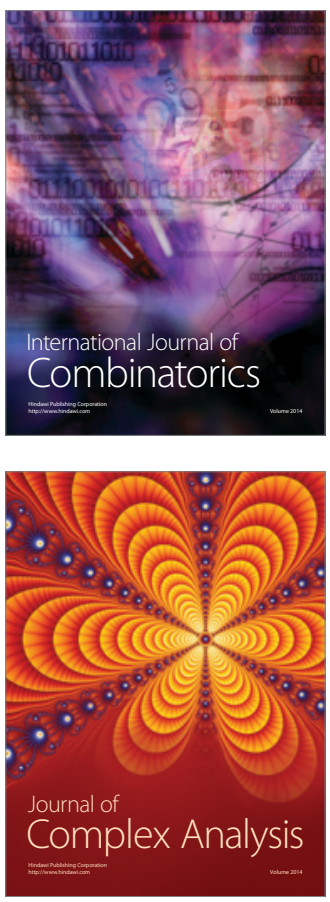

International Journal of

Mathematics and

Mathematical

Sciences
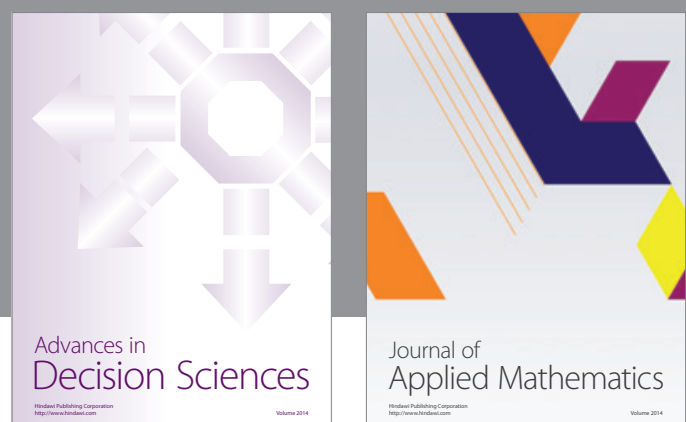

Journal of

Applied Mathematics
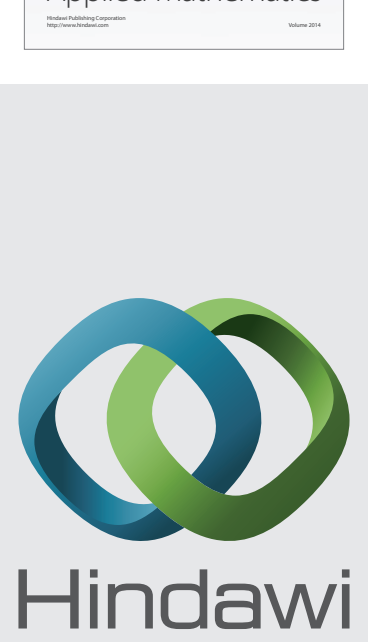

Submit your manuscripts at http://www.hindawi.com
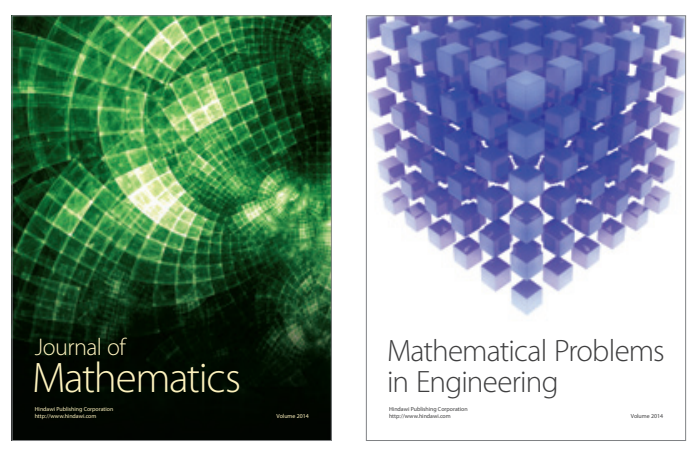

Mathematical Problems in Engineering
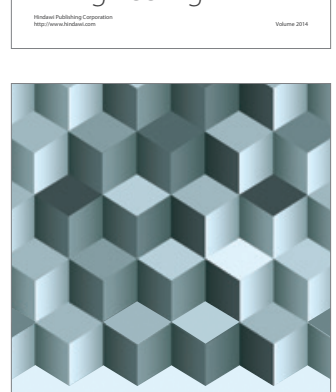

Journal of

Function Spaces
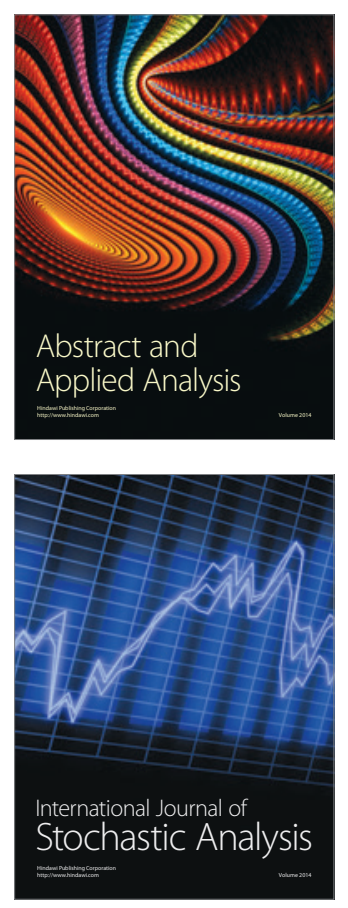

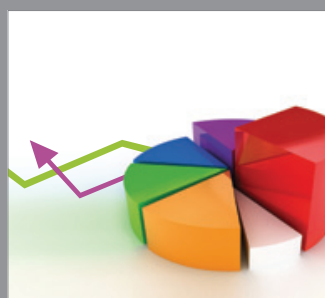

ournal of

Probability and Statistics

Promensencen
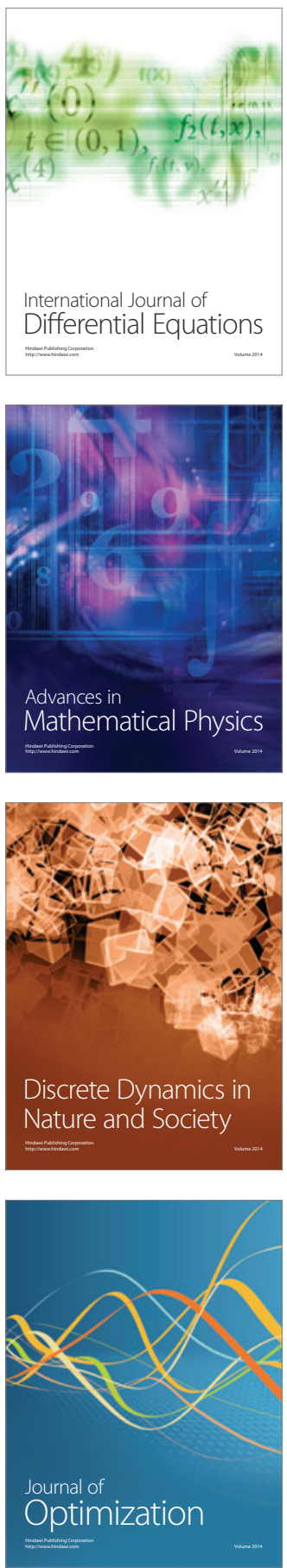